\title{
THREE FATAL CASES OF THE BULBAR TYPE OF POLIOMYELITIS.
}

\author{
BY \\ W. G. SCOTT BROWN, LoNDON.
}

IN the course of an investigation on an epidemic of brainstem poliomyelitis reported in a previous paper, a search was made for similar though fatal cases in order that the pathology might be studied. The three now reported were cases of poliomyelitis of the brainstem type without any spinal involvement and were the total outcome of the search. One case comes from the notes of the Hospital for Sick Children, Great Ormond Street; one from the records of a Metropolitan Asylums Board Hospital; and the third from the Pathological Department of the National Hospital, Queen Square, the case having been treated privately.

\section{EPIDEMIOLOGY.}

It is of interest that in two of these cases the patients had tonsillectomy performed shortly before the onset of symptoms of poliomyelitis. The first patient had enucleation of tonsils performed at Great Ormond Street Children's Hospital 14 days before her admission to the same hospital; she died there on the following day and the diagnosis of recent acute bulbar poliomyelitis was confirmed by histological methods. The first symptoms appeared 11 days after tonsillectomy. In Case 2, prodromal symptoms of poliomyelitis developed 15 days after tonsil enucleation, and a cranial nerve paralysis on the next day. Histological evidence proved the condition to have been poliomyelitis of the brainstem type.

Aycock and Luther (1929), investigating 1,224 cases of poliomyelitis, found that 16 had developed the disease within one month of an operation for removal of the tonsils, and that all of these occurred between the seventh and the sixteenth days inclusive.

It would appear that the operation of tonsillectomy gives an opportunity for the virus, if previously present, to assume its neuropathic tendencies or to gain access to the nervous tissues by implantation at the time of operation or afterwards. It is suggested that there must be some natural barrier in the pharynx which normally wards off the infection before the central nervous system is invaded, and this would account for the fact that pharyngitis is present in a great number of cases. Latent immunity is undoubtedly conferred by this pharyngitis without a spread of the infection to the central nervous system (Fairbrother and Scott Brown, 1931). 
Two of these collected cases confirm Aycock's findings and make it probable that the incubation period of poliomyelitis is between seven and 16 days.

\section{GLINICAL SUMMARY.}

In each case the onset of the illness was sudden, being associated with a sore throat, cough, a raised temperature to about $101^{\circ} \mathrm{F}$. and a correspondingly increased pulse-rate. Vomiting was present in all the cases and was one of the earliest symptoms in two.

Symptoms due to involvement of the central nervous system.Headache was complained of in one case on the day of onset, otherwise it was not a marked feature. There was no head retraction or stiffness of the muscles of the neck in any. In Case 2 spasticity of the upper extremities, with loss of reflexes in the lower, occurred; and in Case 3 loss of reflexes in upper and lower extremities as a terminal sign. There was no paralysis of the upper or lower extremity in any case. Tremor of the fingers was not observed.

Symptoms due to involvement of the cranial nerve nuclei.-In two cases signs of involvement of cranial nerve nuclei occurred on the third and fourth days of the illness respectively.

III, IV, VI. In Case 2 ptosis and internal strabismus were observed on the day the patient died and were probably due to involvement of the third and sixth nuclei.

V. In this case, also, trismus due to irritation of the fifth nucleus was present, and was the first sign of nerve involvement.

VII. Facial paralysis occurred as a terminal event in Case 2.

IX, X. These nuclei are considered together as both are probably concerned to some extent in the production of a nasal voice, dysphagia and choking, which were constant symptoms in these cases.

A palatal paralysis was not observed in spite of the development of a nasal intonation. Dysphagia was associated with choking when swallowing was attempted. The second patient also developed attacks of dyspnœa but laryngeal stridor was not present.

\section{GASE NOTES.}

CASE 1. D. D., age $2 \frac{1}{2}$ years, female.

Two weeks before her admission to Great Ormond Street, tonsillectomy was performed at the hospital. Ailing since, and refused solid food. Four days ago, vomited, was constipated, and had cough. Two days ago, throat appeared to become more sore.

Condition on admission (fourth day of illness) T. 101 ${ }^{\circ}$, P. 144. A well-nourished child, pale, looked ill, irritable when disturbed. On the attempt to give the child fluid, it choked, and was unable to swallow; the palate moved well. Throat generally red and infected. Tonsil area healed, no membrane or ulceration. Central nervous system appeared normal on examination. 
Sixth day of illness. T. $101^{\circ}$, P. 160. General condition appeared worse; cyanosed; cough and choking attacks repeated, with inability to swallow. Palate moved well. A nasal feed caused violent choking and there seemed to be inability to get the food out of the throat. At 6 p.m., collapsed during nasal feed. At 11 p.m., pulse almost imperceptible.

Lumbar puncture was performed and a small quantity of almost pure blood was obtained. Patient died immediately afterwards.

Post-mortem Examination-Macroscopic Appearances.

\begin{tabular}{|c|c|c|c|}
\hline & & & \\
\hline Lungs & $\cdots$ & .. & congestion. \\
\hline Liver & $\ldots$ & $\ldots$ & congestion. \\
\hline Spleen & $\ldots$ & $\ldots$ & normal. \\
\hline Mesenteric & c glands & $\ldots$ & definitely enlarged. \\
\hline Cerebrum & $\ldots$ & $\cdots$ & $\begin{array}{l}\text { natural on outer surface, in the ventricles and } \\
\text { in its substance. }\end{array}$ \\
\hline Cerebellum & ... & $\ldots$ & natural. \\
\hline
\end{tabular}

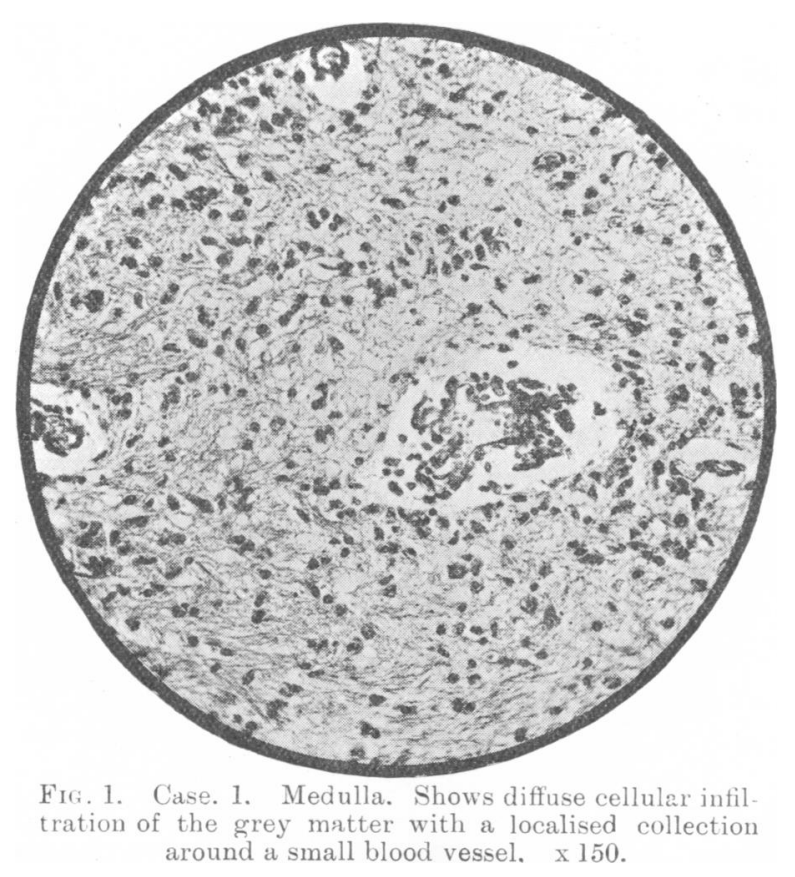

Lying over the anterior surface of the medulla and pons was a thick clot which appeared to be recent. There was no clot on the posterior surface of the medulla but it extended downwards and surrounded the whole cord in the lower dorsal region, making the membranes tense. The clot was inside the pia mater and it stripped off the cord easily. No vessel could be found from which bleeding had taken place and the cord substance appeared normal. Vessels of the medullary region appeared congested. On section small areas of congestion could be seen.

Microscopic Appearances.-Sections of the cord through the lower dorsal region show the surrounding hæmorrhage with congestion of the meningeal vessels but no evidence of poliomyelitis in the grey matter. Sections across the medulla at the level of the middle of the olivary body show histological changes typical of poliomyelitis. There is a diffuse infiltration throughout the grey 
matter though limited to it; more localised and intense infiltration is seen about the nucleus ambiguus, the nucleus of the spinal root of the fifth, and the nucleus of the fasciculus solitarius. Perivascular infiltration, and all stages of nerve degeneration and absorbtion by neuronophagic cells are well seen. The infiltration is mainly lymphocytic though a certain number of polynuclear cells are to be found round the vessels (figs. 1 and 2).

CASE 2. Mrs. D., age 35, married.

During the summer of 1929 was unfit and had a slight temperature, with sore throat. October 11: Tonsils enucleated; patient progressed normally though slowly up to October 26, when she was taking ordinary food. Temperature was

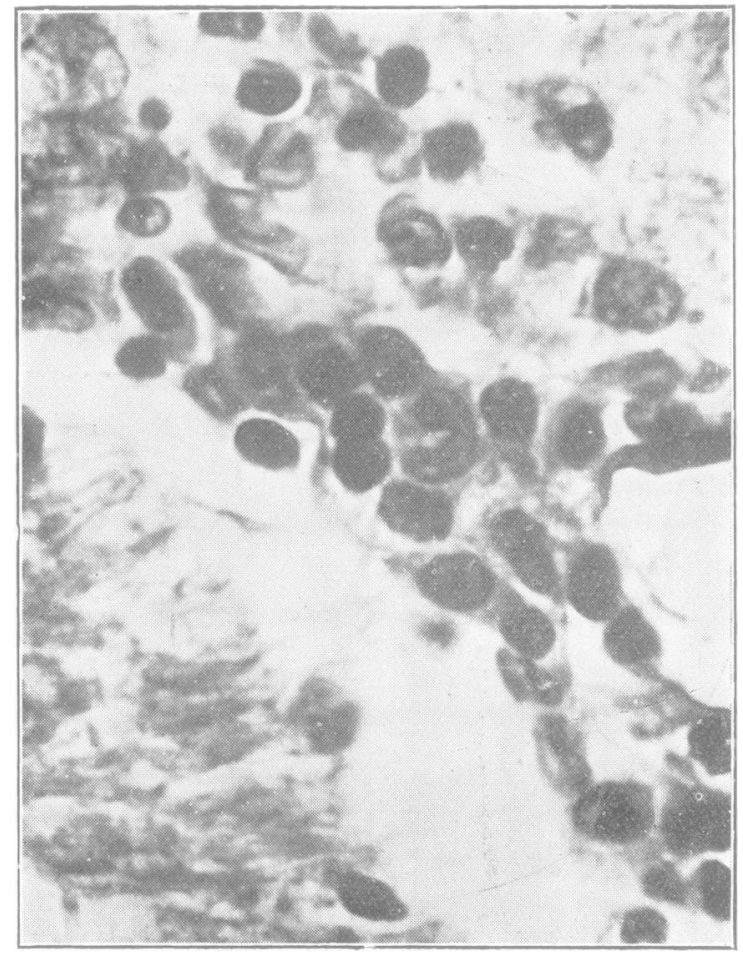

Fig. 2. Case 1. Medulla. An enlargement to show that cells in the perivascular space are mainly lymphocytes with a few polymorphonuclear cells. $\times 750$.

still occasionally $99^{\circ}$. October 27 : Did not feel well, did not want food, vomited. T. $101^{\circ}$. Later in the same day, had a feeling of 'something in her throat' which made swallowing uncomfortable, giving rise to a feeling of rawness.

Somewhat later, developed marked trismus, with tonic contraction of masseter muscles. Actual difficulty in swallowing occurred, and a choking attack in which breathing, too, seemed difficult. 10 p.m.: T. 100:2 ${ }^{\circ}$. Mouth opened half an inch. Speech clear. Tongue clean. Palate moved well. Pharynx could not be seen, as when the tongue was touched the patient immediately retched and vomited. This started an attack of dyspnca, but without laryngeal stridor. There was much frothy saliva in the throat and the difficulty appeared to be more 
pharyngeal than laryngeal. Shortly after, she regurgitated tea through the nose, and the voice became nasal in character. The pharynx was also seen to be dusky and red, with muco-pus running down from above. Digital examina. tion of the pharynx detected no swelling and the manipulation made her easier and made the jaw move better. Nothing else abnormal was discovered in the central nervous system, or in any other system. October 28: After a fairly comfortable night she became worse at noon. Facial weakness developed, also internal strabismus associated with ptosis on one side. Some rigidity of arms and loss of tendon reflexes in both legs. Death occurred the same day.

Post-mortcm Examination. $\rightarrow$ Macroscopic Appcarances.-Nothing abnormal is recorded.

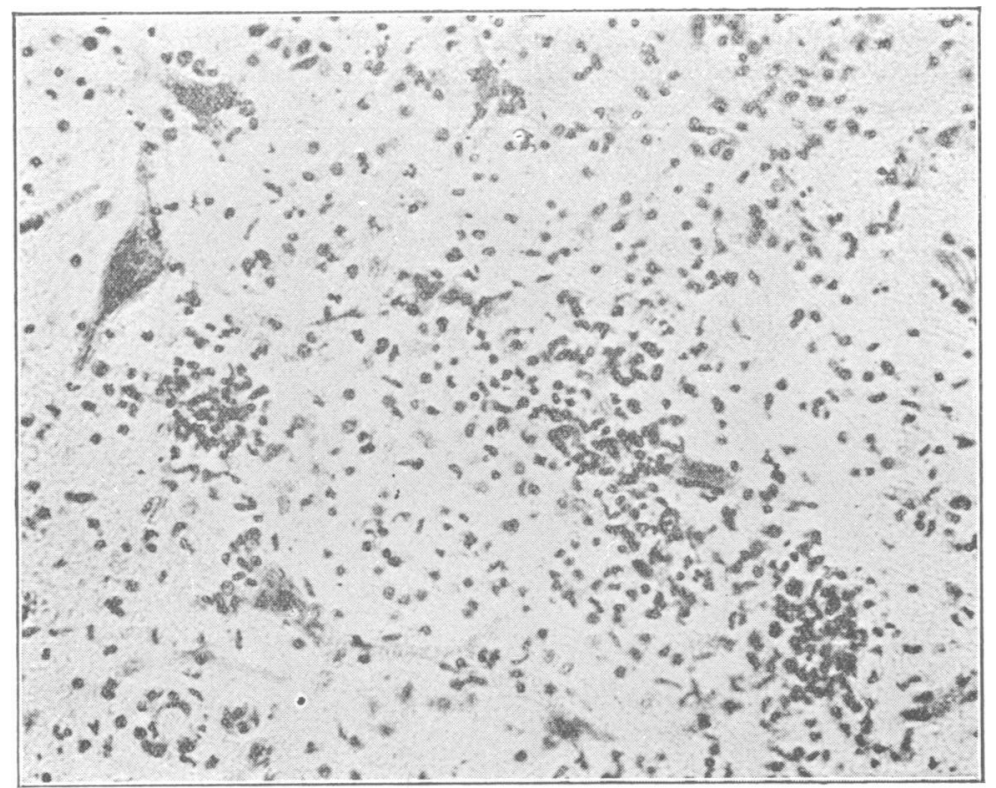

Fig. 3. Case 2. Medulla. Diffuse infiltration of the grey matter with localised collections round cells of the twelfth nucleus. Marked cell destruction.

Microscopic Appearances.-The characteristic changes of poliomyelitis are seen throughout serial sections from the mid-cervical region to the uppermost part of the pons. Section through C 5: Diffuse lymphocytic infiltration, not very intense, limited to the anterior horns is seen. Perivascular infiltration is not marked. Neurolysis and neuronophagia are present. Medulla at decussation of the pyramids: Diffuse infiltration of the upper part of the anterior horn and the region of the descending root of the fifth. Perivascular infiltration is marked. Neurolysis is present. Medulla below the calamus scriptorius: Centred round the calamus is a diffuse infiltration affecting many nuclei. The lowest part of the twelfth nucleus shows infiltration, neurolysis and neuronophagia. Medulla at the tip of calamus scriptorius: The main body of the twelfth nucleus is seriously involved. Intense infiltration and cell destruction are seen in the region of the dorsal nucleus of the vagus on one side: the nucleus ambiguus is also affected. 
The olives are absolutely intact. Upper part of the medulla through fourth ventricle: Diffuse infiltration seen under the floor of the ventricle, with patchy collections not limited to the nuclei. The upper part of the twelfth nucleus is affected and collections are also seen in Deiters' nucleus. The olives and pyramids are free from infiltration. Lower pons: The pyramids and nuclei pontis are quite clear. The region of the ventricle and the sixth and seventh nuclei show infiltration and cell destruction. The main motor nucleus of the fifth nerve is much infiltrated on one side. Upper pons above the middle peduncle: Substantia ferruginea and the ascending sensory root of the fifth nerve show marked infiltration. Upper pons, almost midbrain: Ascending root of the fifth nerve is the only part to show infiltration. The fourth nucleus is clear (figs. 3-5).

CaSe 3. S. C., age $17 \frac{1}{2}$, female.

Patient was taken ill on September 12, 1929, complaining of headache, pains all over the body, and slight sore throat. Previously she had been in good health

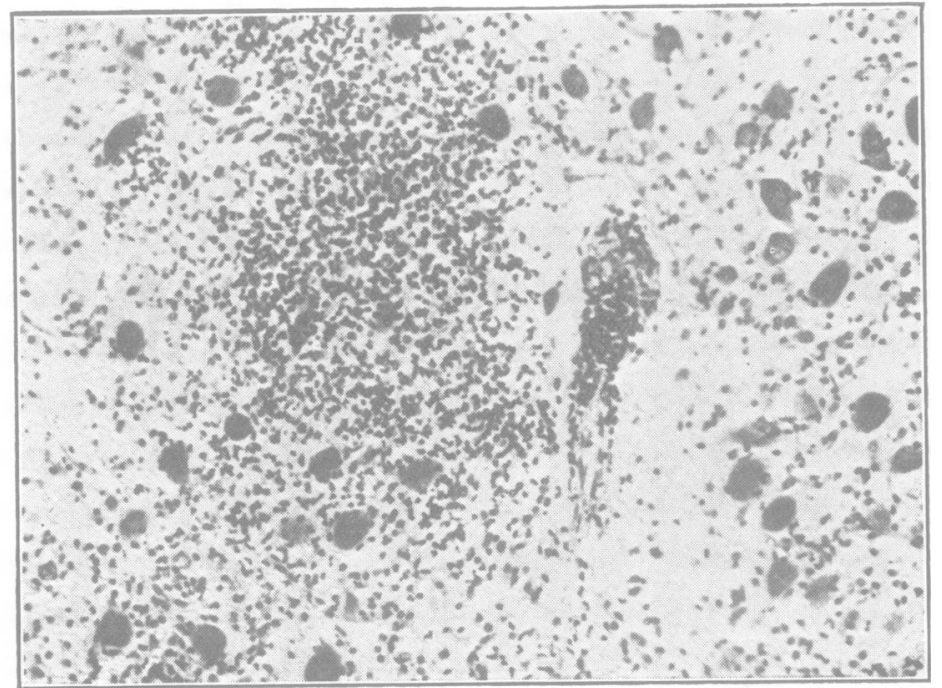

Fi(i. 4. Case 2. Pons. The motor nucleus of the fifth nerve show's most intense intiltration, with marked neurolysis and neuronophagia.

She felt better the next two days, but on September 15 had to return to bed and the doctor was called in. Had several attacks of vomiting and coughing. four days later, was unable to swallow and was admitted to hospital.

Condition on admission (seventh day of illness).-Patient was apathetic and complained of general weakness. She was unable to swallow and attacks of choking followed each attempt: a large amount of mucus had collected in the pharynx. Palatal movements were normal. There was paresis in the upper and lower extremities. Knee-jerks were present but sluggish. Abdominal reflexes were absent. There was no sensory disturbance and no ocular paralysis. Pupils were equal and reacted to light. No head retraction was observed. The heart and lungs were normal.

September 21 : Rather more drowsy but answered questions intelligently when roused. Inability to swallow persists, with choking caused by mucus in the pharynx. 
September 23: Arm and leg reflexes not elicited, but can move limbs voluntarily. Otherwise the symptoms continue as before.

September 24: Died at 2.50 a.m. on the twelfth day of the illness.

Examination of the cerebrospinal fluid.-Slightly under pressure, clear, watery, no clot. Cells : 2 lymphocytes per c.mm. No organisms seen. No tubercle bacilli. Culture showed no growth. Chlorides : 0.75 per cent. Sugar: a trace. Albumin : 0.025 per cent. Globulin : nil.

Post-mortem Examination.-Surface of brain, cord, and meninges was normal. Congestion of the grey matter in the cervical region with small punctate hæmorrhages in the anterior horns. The brainstem showed a similar congestion.

Microscopic Appearances.-Section through cervical cord.-The grey matter of the anterior horns shows diffuse and intense infiltration, with some localised

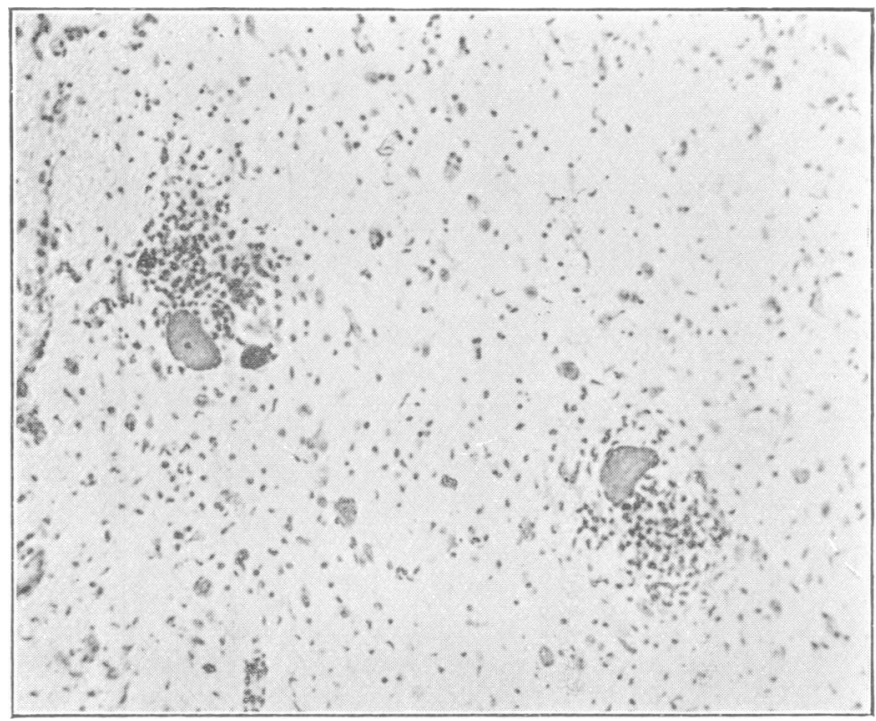

FIs. 5. Case 2. Junction of pons and midbrain. Highest level at which infiltration is seen. It occurs round the cells of the mesencephalic root of the fifth nerve and is the only evidence of inflammation at this level. The oculomotor nuclei are not involved.

collections of cells round nerve-cells. Perivascular infiltration with lymphocytes occurs, some polynuclear cells being present among them.

Section through the mid-pons.-A diffuse infiltration is present beneath the floor of the fourth ventricle with more intense infiltration around the nuclei. Laterally, the fifth nucleus is very definitely invaded and cell degeneration or absorbtion by neuronophagic cells is a marked feature. Perivascular infiltration is seen. The trapezium, pyramidal bundles and nuclei pontis are not involved (fig. 6).

\section{PATHOLOGY.}

Morbid Anatomy.-In the nervous system, the surfaces of the brain, cord and meninges appear normal, cr slightly congested. In Case 1 there 
was hæmorrhage over the anterior surface of the pons and medulla and over the whole circumference of the cord at a lower level. The hæmorrhage was under the pia mater and appeared to arise from a vessel on the anterior surface of the medulla. On section of the cord at the affected level, the grey matter of the anterior horns appeared hyperæmic. This stage is well illustrated in Case 3. Hypertrophy of lymphoid tissue was seen in Case 1 where there was definite enlargement of the mesenteric glands.

II istology.-The meninges are seen to be congested and are the site of a small round-celled infiltration. The hæmorrhage round the cord in Case 1

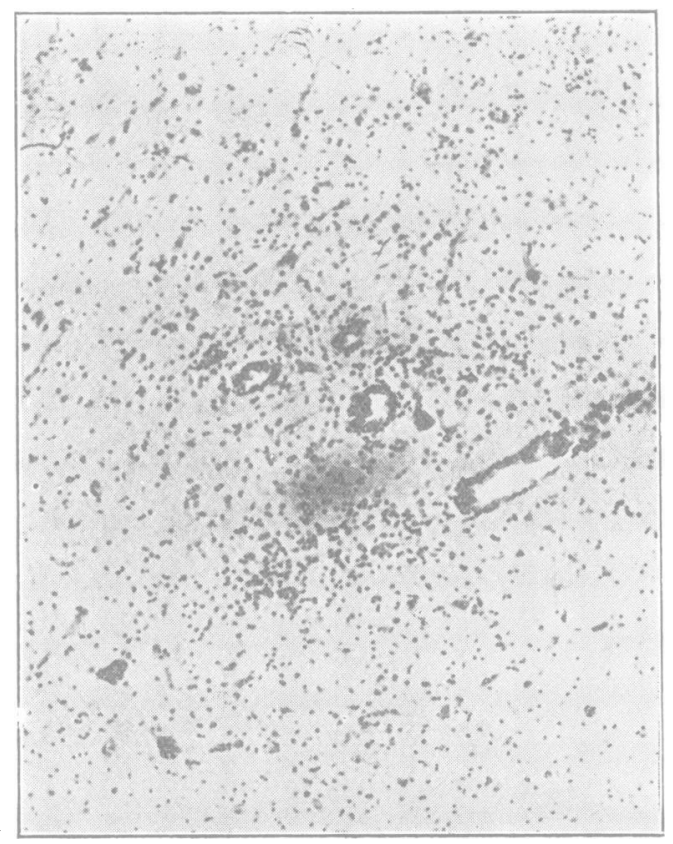

Fic. 6. Case 3. Pons shows diffuse and perivascular infiltration.

is probably due to the intensity of the congestion which has caused a rupture of small vessels.

The main histological changes are found in the grey matter of the bulb, and consist of a diffuse cellular infiltration with a localized collection about the vessels, and degeneration and phagocytosis of the nerve-cells. The infiltrating cells are mainly lymphocytes which are scattered diffusely through the grey matter, or are collected in groups around an effete nervecell. The localized perivascular infiltration is in the adventitial lymphatic spaces and gives rise to the histological appearance described as ' cuffing' 
of the vessel. The cells are again mainly lymphocytes, but there are among these cells a number of polymorphonuclear cells. Many nerve-cells show chromatolysis due probably to the direct action of the virus and in some cases this degeneration has taken place before the appearance of perivascular cuffing. Some of these damaged cells are surrounded by groups of lymphocytes, some are invaded by the glial neuronophagic cells, and others entirely replaced by them. Small hæmorrhages with areas of degeneration are visible in the grey matter.

In Case 2 it will be seen that a number of the cranial nerve nuclei are involved, but there is practically no spread outside the grey matter. In spite of the extent of the lesions in the pontine and bulbar nuclei, the pyramidal tracts have escaped. It is of interest to note that in the highest section in Case 2, viz., through the junction of pons and midbrain, the only area involved is that of the mesencephalic root of the fifth nerve.

\section{DISCUSSION.}

This histological picture and account of the morbid anatomy are in agreement with the accepted pathology of poliomyelitis as established by Harbitz and Sheal (1907) and Wickman (1911). Much discussion has centred round the origin of the various cells in the lesions of poliomyelitis, but it is generally agreed that the infiltrating cells are mainly microglia and lymphocytes, though a number of polynuclear cells are also present in the perivascular infiltration. Plasma and plasmoid cells are seen but rarely.

Infiltration is seen round the mesencephalic root of the fifth nerve in the section at the junction of the pons and midbrain in Case 2 , but there is no infiltration or involvement of the fourth nucleus, or any other nucleus or tract at this level. This fact supports the evidence of Fairbrother and Hurst (1930), that the spread of the virus is by the axis-cylinders. Goodpasture (1925) had shown previously that infiltration appeared round the nucleus of the fifth nerve after the virus was injected into the cheek.

Although the virus almost certainly spreads by the axis-cylinders of the pyramidal tracts to the anterior horn-cells no infiltration of the pyramidal tracts is seen, indicating that this tissue has no affinity for the virus. The reaction of the virus and tissue where such affinity exists probably originates some metabolic or chemical change which produces chemiotaxis of wandering cells and in this way the infiltration.

The histology of the brain-stem type of poliomyelitis is slown in this paper to be similar to that of the spinal form, and the microphotographs indicate that the bulbar lesions may be quite as intense as those in the spinal cord. This histological picture can be distinguished from that ef epidemir 
encephalitis, and the more recently described meningo-encephalo-myelitis following the specific fevers or vaccination. In epidemic encephalitis, the lesions are seen mainly in the basal ganglia and midbrain, less in the medulla and cord. The meninges are scarcely involved. The intense and often wide ring of ' cuffing' is most characteristic, though polymorphonuclear cells and the diffuse infiltration of poliomyelitis are absent. Neuronophagia is rare. In meningo-encephalo-myelitis the white matter of the brain is more affected than the grey matter, whereas in the cord both are equally affected. The meninges escape involvement. The lesions are perivascular but they extend outside the sheath, giving rise to a characteristic zone of well-marked demyelination of nerve fibres.

McIntosh and Scarff (1928) state that in poliomyelitis lesions are mainly found in the grey matter of the cord, while changes in the brain are very rare and relatively slight. Undoubtedly every part of the central nervous system may be affected; lesions have been found in the cortex, midbrain, basal nuclei and peripheral nerves, as well as in the brain-stem and cord; but on searching for cases of ' cerebral' poliomyelitis one is struck by the increasing scarcity of these cases since 1918, when encephalitis lethargica was first described by von Economo. Ayer (1928) does not consider an encephalitic form, and Silverman (1929) says that, among 266 cases, one case only was suggestive of the cerebral or encephalitic type and that the evidence was by no means convincing. Experimental work shows that even when the active virus is inoculated into the occipito-parietal lobe of a monkey, it is almost invariably the opposite lumbar cord that is first affected and the local cerebral condition at any time is not very marked. Thus there is considerable doubt as to the existence of a cerebral or a cerebellar type as a distinct form of the disease.

It was thought that the virus spread by a meningeal route, but Hurst (1929) described the result of histological examination of serial sections of the brain in monkeys killed on each day from the first to the fifth, following intracerebral inoculation, and demonstrated an overflow of inflammatory cells from the deeper infiltrated vessels along the perivascular spaces to the meninges. A meningeal lesion therefore complicates a deeper lesion.

Hence, three of the groups in Wickman's classification exist only doubtfully, viz., the cerebral, cerebellar, and meningitic groups, and Batten (1916) disposes of yet another, the neuritic group.

As there appears to be ample justification for considering the bulbar groun of cases as a definite trpe, the old classification should be replaced by the simple division of cases into spinal, bulbar and abortive. 


\section{SUMMARY.}

1. A relationship between tonsillectomy and the onset of poliomyelitis is discussed, with its bearing on a probable incubation period.

2. Clinically the cases show a prodromal period with a raised temperature and pulse, pharyngitis and vomiting. This is followed by involvement of the cranial nerves, with resulting paralyses.

3. These fatal cases are proved to be poliomyelitis by pathological examination. Evidence is brought forward in favour of the theory of axon spread of the virus, and a simplified classification is adopted to accommodate recent histological and clinical views as to the site and type of lesions occurring in the central nervous system.

My thanks are due to Dr. Poynton, Dr. Ryle, Dr. Greenfield and Dr. Weston Hurst for permission to publish these cases. 